Mechanism of field-induced unwinding of $\mathrm{Sm} C^{*}$ helix and bias field dependencies of dielectric permittivity and effective polarization

This article has been downloaded from IOPscience. Please scroll down to see the full text article.

2008 EPL 8226003

(http://iopscience.iop.org/0295-5075/82/2/26003)

View the table of contents for this issue, or go to the journal homepage for more

Download details:

IP Address: 134.226.1.229

The article was downloaded on 01/07/2010 at 11:46

Please note that terms and conditions apply. 


\title{
Mechanism of field-induced unwinding of SmC* helix and bias field dependencies of dielectric permittivity and effective polarization
}

\author{
JANG-Kun Song, U. MAnnA and J. K. ViJ (a) \\ Department of Electronic and Electrical Engineering, Trinity College, University of Dublin - Dublin 2, Ireland, EU
}

received 24 January 2008; accepted in final form 29 February 2008

published online 28 March 2008

PACS $61.30 . \mathrm{Dk}$ - Continuum models and theories of liquid crystal structure

PACS 61.72.Lk - Linear defects: dislocations, disclinations

PACS 77.84.Nh - Liquids, emulsions, and suspensions; liquid crystals

\begin{abstract}
We propose an unwinding model based on helical fractures confirmed by spectroscopic and microscopic observations. This model combined with the fixed helical pitch can explain the various physical phenomena observed in $\mathrm{Sm}^{*}$ phase of ferroelectric liquid crystals. These are the dc field dependencies of i) dielectric strength, ii) relaxation frequency of the Goldstone mode, and iii) the effective polarization. The conventional diverging pitch model where the helical pitch increases with an increase in the field is shown not to satisfactorily explain these observations.
\end{abstract}

Copyright (C) EPLA, 2008

The mechanism of unwinding of a helical structure is common to many areas in soft condensed matter physics and in biology. The super twisted helix of DNA is unwound with the addition of enzymes called topoisomerases which fracture the helix selectively, and then reconnects it for duplication and reproduction [1]. The break in the helical structure into segments is an easier and an efficient method for solving a topological problem caused by the unwinding, i.e. the phase difference of $2 \pi m$ between the two ends of the helix ( $m$ denotes the number of nodes including zero). Without this break, the end molecules of the helix must make numerous turns for an unwinding process to be completed. The helical unwinding in FLCs brought about by the application of an external electric field has a similar problem as that of DNA, but has been solved differently. In 1977, Meyer [2] gave a model for the field-induced helical unwinding process in $\mathrm{SmC}^{*}$, analogous to that in cholesteric liquid crystals also proposed by him [3]. The continuity of directors along the normal to layers is not broken during the unwinding process because the unwinding occurs through the pitch diverging, that is, the pitch increases with an increase in the applied field and finally it diverges to infinity at the critical field for completely unwound state $E_{c}[4-8]$. This is called the diverging pitch model and is based on the continuum approximation, where the elastic energy is proportional to the square of the deformation of directors, hence the deformation by an

\footnotetext{
(a) E-mail: jvij@tcd.ie
}

external force should be spatially continuous for minimizing the elastic energy. However, we showed recently that a number of helical fractures are produced by a relatively low field in some FLCs [9]. This is to state that the continuity of the helix can easily be broken, and a number of helical fractures occur. The helical fracture means that the angle difference $\left(\Delta \phi_{i, i+1}\right)$ between the $c$ directors of the two adjacent layers experiences a large change whereas the other angle differences stay small. This is fundamentally different from the diverging pitch model and the continuum approximation. Note that the helical fracture requires too large elastic energy to appear in the continuum approximation. However, it is well known that in the field-induced switching of antiferroelectric phase to ferroelectric state, $\Delta \phi_{i, i+1}$ changes easily from 0 to $\pi$ by moderate electric fields through a non-homogeneous process, i.e. the solitary-wave propagation [10]. This implies that the fractures between the smectic layers could occur through a non-homogeneous process. Thus, the helical unwinding in FLCs can be based either on the continuum theory or on a theory where the helix is fractured into smaller segments.

This letter proposes the theory for the helical unwinding of $\mathrm{Sm} C^{*}$ phase based on the model where the topological problem of the helical unwinding is solved by helical fractures, and explains the experimental observations of dielectric response as a function of the bias field. This cannot be explained satisfactorily by the diverging pitch model. 

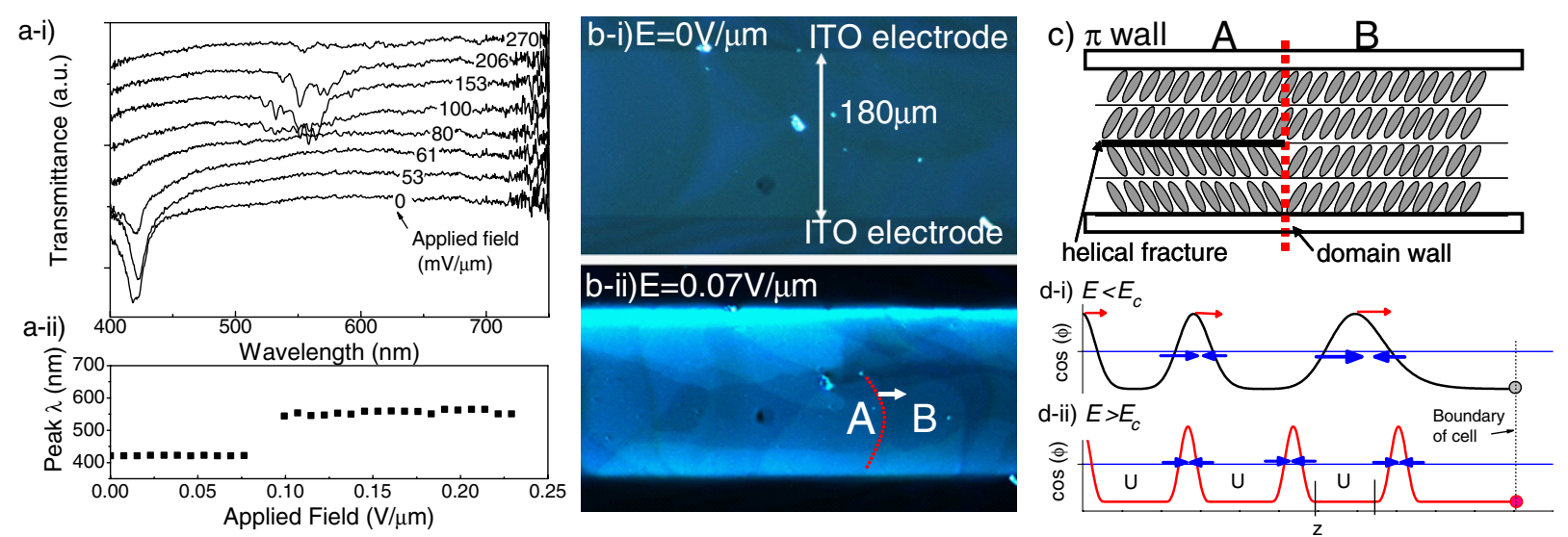

Fig. 1: (a) Visible spectroscopy (a-i) and the selective reflection peak (a-ii) with increasing fields for a thick homeotropic cell $(d \approx 100 \mu \mathrm{m}$, space between two metal wall electrodes $\approx 500 \mu \mathrm{m})$. It shows a step-like increase at $0.07 \sim 0.1 \mathrm{~V} / \mu \mathrm{m}$. (b) Microscopy for a thin homeotropic cell $\left(d \approx 7 \mu \mathrm{m}, 88^{\circ} \mathrm{C}\right)$. From the field where the step-like increase of the peak occurs, many domains indicating the helical fractures appear and propagate. The boundary between A and B moves along the arrow. (c) Schematics for the helical fractures shown for a homeotropic cell, $\pi$ walls always are accompanied by discrete $\mathbf{c}$ directors, that is, helical fractures. For example, A and B are separated by a $\pi$ wall, and A area has the fractured plane between the two layers. The fractured plane may reunite by an additional propagating wall at the same layers. (d) Schematics for the diverging force of the pitch middle line as zero value of $\cos (\phi)$. In the diverging pitch model, when $E<E_{c}(\mathrm{~d}-\mathrm{i})$, the unbalanced elastic forces at $2 \pi$ solitons makes the elastically connected $2 \pi$ solitons diverging to the cell boundary, but when $E>E_{c}$ (d-ii), the unwound parts between $2 \pi$ solitons isolates $2 \pi$ solitons and no diverging force exists. (Note that the $2 \pi$ solitons along normal to layer shown in (d-ii) is different from the $\pi$ walls along the layers in (b,c).)

Recently, Krueger et al. [11] reported that the helical pitch in various FLC materials does not change during the helical unwinding in planar cells in contrast to a few earlier observations [12] on DOBAMBC and similar compounds. They explained the phenomenon by using earlier models where the surface effect with disclination lines on surface is large enough to prevent helix from diverging [13], and that the relaxation frequency of the helical unwinding is too low to be detected in the usual experiments [6]. In a thin cell using a long helical pitch material, the helical structure is suppressed by the surface effect, and a deformed helical structure appears in the center of the cell. The unwinding process in this cell is rather easily explained by the model suggested by Glogarova et al. [13], where the surfaceinduced unwound part expands with increasing field. However, an intrinsic bulk helical unwinding process in a thick planar cell having short helical pitch or in a homeotropic cell is not understood clearly.

We measured the selective reflection peak using 12OF1M7 (Kingston Chemicals Hull, UK) and a thick homeotropic cell $(d \approx 100 \mu \mathrm{m})$, where the surface effect is minimized. Surprisingly, the selective reflection peak $\lambda(p \approx \lambda / n, n$ is a refractive index) shows a step-like increase at $0.07 \sim 0.1 \mathrm{~V} / \mu \mathrm{m}$ (fig. $1(\mathrm{a}-\mathrm{ii})$ ), and at this range of fields, the peak was not observed. At large fields, the broad and multi-peaks reappeared, and disappeared with a further increase in the field (fig. 1(a-i)). Microscopic observation with increasing fields using a thin homeotropic cell $(d \approx 7 \mu \mathrm{m})$ shows that many domains occur for $E \approx 0.07 \mathrm{~V} / \mu \mathrm{m}$ as shown in fig. 1(b). The electric field for the emergence of the domain walls corresponds exactly to the step-like increase of the selective reflection peak. The domain walls have single sharp lines and propagate independently of each other indicating that these are $\pi$ walls propagating along layers [9]. $\pi$ walls always accompany with the helical fractures as shown in fig. 1(c) (see caption). As the walls propagate, the fracture area enlarges. This observation clearly shows that the helical unwinding occurs through the helical fractures. The applied field increases the stress on the helix, and the stress is released by the helix getting fractured through the domain wall propagation. Here, the helical pitch also increases with the helical fractures, because each $\pi$ wall propagation reduces the helix by about half a turn. This is fundamentally different from a continuous increase in the helical pitch expected from the diverging pitch model.

The helical fracture with propagating solitary wave is a non-homogeneous process, and it is difficult to express the free energy of the cell governing the unwinding process exactly. Moreover, the mechanism of the emergence of the fractures is not clear at this stage, though a possible model was suggested in our previous paper based on a special steric energy [9]. However, a break in the helix gives rise to an additional flexibility to explain various physical properties occurring during the unwinding. We can introduce a completely unwound part (U) between the highly deformed c directors along normal to the layers called as $2 \pi$ soliton [4] as shown in fig. 1(d). Note that the completely unwound part (U) cannot exist in the diverging pitch model, because it makes the soliton isolate and it prevents the pitch from diverging. That is, the isolated soliton cannot have a diverging force normal 
to the applied field, because the diverging force arises from the unbalanced elastic forces of the $2 \pi$ soliton so as to reduce the total elastic energy (fig. $1(\mathrm{~d}-\mathrm{i})$ ), but the horizontal shift of the isolated $2 \pi$ wall does not reduce the total free energy (fig. 1(d-ii)). Urbanc et al.'s rigorous calculation [6] based on the diverging pitch model shows that the relaxation frequency of the pitch divergence converges to zero at $E_{c}$, which means that the diverging force disappears when $E>E_{c}$.

Using the diverging pitch model, the dielectric response of the $\mathrm{SmC}^{*}$ phase with dc bias voltage was already studied by Urbanc et al. $[5,6]$. However, the theoretical prediction does not directly accord with the experimental results such as the dielectric strength $\left(\Delta \epsilon_{G}\right)$ and the relaxation frequency $\left(f_{G}\right)$ for the Goldstone mode and the effective polarization along electric field $\left(\left\langle P_{E}\right\rangle\right)$. These properties have theoretically been predicted to have a sudden change near $E_{c}$ as shown later in fig. 3. However, all the experimental results [13-18] given so far have shown that these parameters do change gradually and finally reach saturation. For the bias dc field dependencies of $\Delta \epsilon_{G}$ and $f_{G}$, a threshold voltage at a much lower field than $E_{c}$ has been observed experimentally [18], which cannot be explained by the diverging pitch model. Moreover, $f_{G}$ in $\mathrm{SmC}^{*}$ is observed to increase with $\mathrm{dc}$ bias field [13-18], but it is expected to decrease with increasing pitch in the diverging pitch model. Pfeiffer et al. [14] suggested that the phenomenon may arise from a nonlinear elastic constant, that is, the elastic constant increases with increasing deformation as is in a practical elastomer. However, according to the Landau expansion for the free energy in $\mathrm{Sm} C^{*}$, the elastic constant with angular distortion will decrease rather than increase for a larger angular distortion [10,19].

Here, we explain the experimental observations based on the non-diverging pitch model. We fix the pitch independent of the bias field and we consider the completely unwound parts between the highly deformed parts. We start from the well-known free-energy expression and the corresponding Euler-Lagrange equation in the static case [2]:

$$
\begin{aligned}
& f=\int\left[\frac{K}{2}\left(\frac{\mathrm{d} \phi}{\mathrm{d} z}-q\right)^{2}-P_{s} E \cos \phi\right] \mathrm{d} z, \\
& K \frac{\mathrm{d}^{2} \phi}{\mathrm{d} z^{2}}-P_{s} E \sin \phi=0,
\end{aligned}
$$

where the wave vector $q(=2 \pi / p$, where $p$ is the pitch $)$ is adopted to produce a helical structure at zero field.

We derive an analytical expression using the assumption of the fixed pitch and introducing the completely unwound part in the helix. Using the Fourier expansion, we write the expression for the dependence of the azimuthal angle as a function of the distance along the layer normal $\phi(z)=$ $q z+\sum \phi_{i} \sin [(i+1) q z]$, where $i=0,1, \ldots$, and $\phi_{i}$ 's are the Fourier coefficients. To estimate the dielectric strength as function of the applied field, a simplified form of the

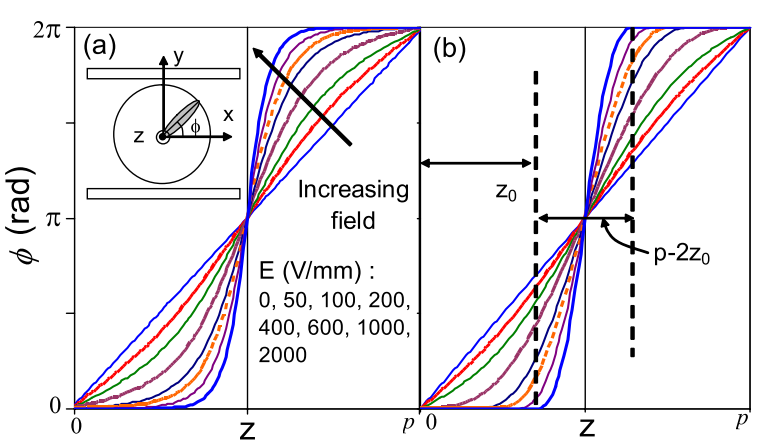

Fig. 2: Schematic and plots for a homogeneous aligned planar cell. Director distributions for different dc fields. (a) simulating eq. (1) directly. (b) Simplified $\phi$ of eqs. (3) and (4); $z_{0}$ and $p-2 z_{0}$ are plotted for $E=2 \mathrm{kV} / \mathrm{mm}$. Simulation conditions: $P_{s}=100 \mathrm{nC} / \mathrm{cm}^{2}, K=10^{-7} \mathrm{dyn}, p=400 \mathrm{~nm}$ and thickness of a single layer $=4 \mathrm{~nm}$. $\phi$ is defined in the inset of part (a).

director distribution function is required. We shall use up to the second term of the Fourier expansion at low fields $\left(E<E_{t}\right)$. At high fields $\left(E>E_{t}\right)$, some part of the helix is unwound completely, and the other part is highly distorted. Therefore, we divide the unwinding process into two stages: low and high fields, separated by a transition field $E_{t}$ :

i) low fields $\left(E<E_{t}\right)$

$$
\phi_{l}(z) \simeq q z-\phi_{0} \sin (q z), \quad \text { where } \phi_{0}<1
$$

ii) high fields $\left(E>E_{t}\right)$

$$
\begin{aligned}
& \phi_{h}(z) \simeq \rho\left(z-z_{0}\right)-\sin \left[\rho\left(z-z_{0}\right)\right], \\
& \quad \text { for } z_{0}<z<\left(p-z_{0}\right), \\
& \phi_{h}(z)=0, \quad \text { for } z<z_{0} \text { or } z>\left(p-z_{0}\right),
\end{aligned}
$$

where $\rho=2 \pi /\left(p-2 z_{0}\right)$, that is, $z_{0}=p / 2-\pi / \rho$.

From eqs. (2), (3) and (4), we can find $\phi_{0}, \rho$ and $E_{t}$ as

$$
\begin{aligned}
\phi_{0} & \simeq \frac{P_{s} E}{K q^{2}}\left[1+\frac{1}{2}\left(\frac{P_{s} E}{K q^{2}}\right)^{2}\right], \\
\rho & \simeq \sqrt{\frac{5 P_{s} E}{4 K}}, \quad E_{t} \simeq \frac{4 K q^{2}}{5 P_{s}} .
\end{aligned}
$$

Surprisingly, $E_{t}$ is found to have similar dependencies on the various parameters as $E_{c}=\pi^{2} K q^{2} /\left(16 P_{s}\right)$ obtained by Meyer [2]. This will be discussed later.

To verify the simplified director distribution functions eqs. (3) and (4), we compare the two director distributions by simulating from the free energy eq. (1) and by simulating the simplified eqs. (3) and (4). As shown in fig. 2, two distributions agree with each other. This confirms the validity of the simplified distributions.

Corresponding to eq. (1), the dynamic Euler-Lagrange equation for the motion of the $\mathbf{c}$ director by a weak ac field is written as

$$
\gamma \frac{\partial \phi}{\partial t}-K \frac{\partial^{2} \phi}{\partial z^{2}}+P_{s} E \sin \phi=0
$$


where $\gamma$ is the viscosity of the medium. The applied field and $\phi_{l}$ under the low-field case are written as

$$
\begin{aligned}
E & =E_{d c}+\delta E=E_{d c}+\delta E_{0} e^{j w t}, \\
\phi_{l}(z) & \simeq q z-\phi_{0} \sin (q z)-\delta \phi_{0} \sin (q z) e^{j w t} .
\end{aligned}
$$

By using eqs. (8), (9) and (7), and the fact that the timedependent and -independent terms should independently be zero, we can obtain $\delta \phi_{0}$ as

$$
\delta \phi_{0}=\frac{\phi_{0} \delta E_{0} / E_{d c}}{1+j w \gamma /\left(K q^{2}\right)} .
$$

The dielectric susceptibility can be obtained,

$$
\chi=\mathrm{d}\left\langle P_{E}\right\rangle / \mathrm{d} E=\partial\left\langle P_{E}\right\rangle / \partial \delta E,
$$

where $P_{E}$ is the component of $P_{s}$ parallel to the applied field, and $P_{E}=P_{s} \cos \phi$.

$$
\begin{aligned}
\left\langle P_{E}\right\rangle_{l}= & \left(P_{s} / 16\right)\left[8 \phi_{0}-\phi_{0}^{3}+\left(8-3 \phi_{0}^{2}\right) \delta \phi_{0} e^{j w t}\right. \\
& \left.-3 \phi_{0} \delta \phi_{0}^{2} e^{j 2 w t}+\ldots\right] .
\end{aligned}
$$

From eqs. (11), (12), and on assuming that $\chi=\left(\epsilon_{0} \Delta \epsilon_{G}\right) /$ $\left[1+j w /\left(2 \pi f_{G}\right)\right]$, we find $\Delta \epsilon_{G, l}$ and $f_{G, l}$ at low fields as

$$
\begin{aligned}
\Delta \epsilon_{G, l} & \simeq \frac{P_{s} A}{32 \epsilon_{0}}\left[16+\frac{2 P_{s}^{2} E_{d c}^{2}}{K^{2} q^{4}}-\frac{9 P_{s}^{4} E_{d c}^{4}}{K^{4} q^{8}}\right], \\
f_{G, l} & =\frac{K q^{2}}{2 \pi \gamma} .
\end{aligned}
$$

For the dielectric response at high fields, we can use eq. (4) for the director distribution. The unwound part of the helix $(\phi=0)$ does not contribute to the dielectric response, so we need only to consider the distorted part. We shall use $z^{\prime} \equiv z-z_{0}$. Then, $\phi$ under high applied fields is written as $\phi_{h}(z) \simeq \rho z^{\prime}-\sin \left(\rho z^{\prime}\right)-\delta \phi_{0} \sin \left(\rho z^{\prime}\right) e^{j w t}$. Following the similar procedure as for low fields, we obtain

$$
\begin{aligned}
\left\langle P_{E}\right\rangle_{h}= & \frac{2 z_{0} P_{s}}{p}+\frac{\left(p-2 z_{0}\right) P_{s}}{p}\left[\frac{7}{16}+\frac{5}{16} \delta \phi_{0} e^{j w t}\right]= \\
& \frac{P_{s}}{16 \rho}\left[16 \rho-9 q+5 q \delta \phi_{0} e^{j w t}\right] \\
\Delta \epsilon_{G, h}= & \frac{5 q P_{s}}{16 \epsilon_{0} \rho E_{d c}}=\sqrt{\frac{5 q^{2} K P_{s}}{64 \epsilon_{0}^{2} E_{d c}^{3}}} \\
f_{G, h}= & \frac{K \rho^{2}}{2 \pi \gamma}=\frac{5 P_{s} E_{d c}}{8 \pi \gamma}
\end{aligned}
$$

In eq. (15) the term $\left(p-2 z_{0}\right) / p$ shows that only the distorted part of the helix can contribute to the dielectric response. Thus, both the dielectric strength and the relaxation frequency are found to depend strongly on the amplitude of the dc bias at large fields. However, the mechanism does not arise from the nonlinearity of $K$, but from an increase in the effective wave vector $\rho$. That is, the high bias field increases $z_{0}$ and reduces the effective pitch $\left(p-2 z_{0}\right)$, which in turn increases the relaxation frequency.

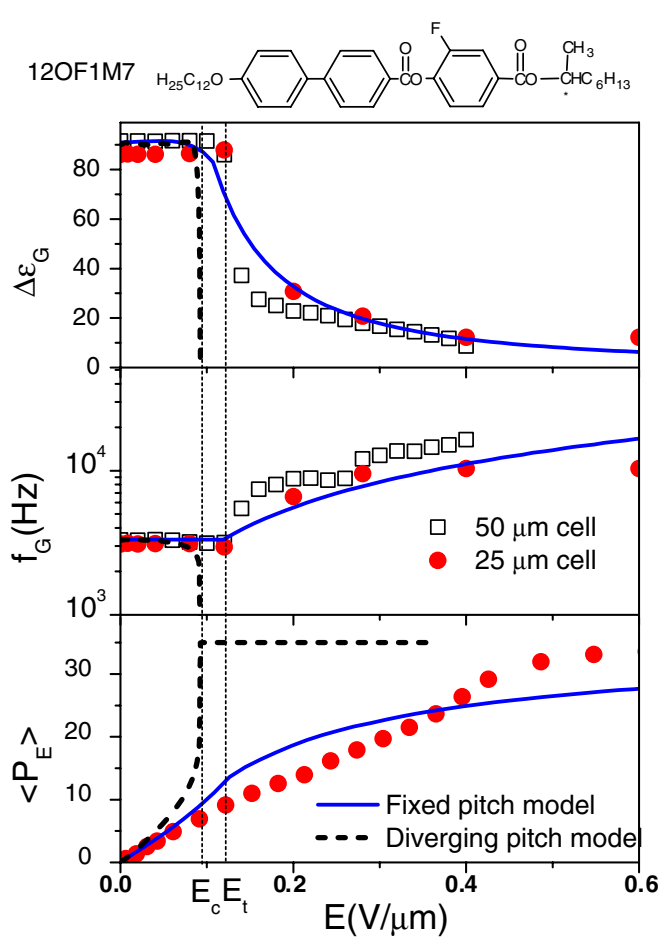

Fig. 3: Experimental data for $50 \mu \mathrm{m}$ (open squares) and $25 \mu \mathrm{m}$ (solid circles) thickness planar cells using 12OF1M7 and simulation results for the fixed and diverging pitch models. (Simulation conditions selected for the best-fitted curves.) Transition temperatures are $\mathrm{Sm}_{A}^{*} 77.2, \mathrm{Sm} C_{\gamma}^{*}$ 80.2, $\mathrm{AF}$ 82.8, $\mathrm{Sm}^{*}$ 91.8, $\operatorname{Sm} A$ 105.7 I (in units ${ }^{\circ} \mathrm{C}$ ).

The dc bias dependencies of $\Delta \epsilon_{G}, f_{G}$ and $\left\langle P_{E}\right\rangle$ are compared with experimental data in fig. $3,\left\langle P_{E}\right\rangle$ is measured using the experimental method (see $[8,20]$ ). Those simulated from the diverging pitch model are also plotted for comparison [5]. $\left\langle P_{E}\right\rangle$ was measured using a $25 \mu \mathrm{m}$ thickness planar cell and $12 \mathrm{OF} 1 \mathrm{M} 7$ at a temperature $2{ }^{\circ} \mathrm{C}$ below from $\mathrm{Sm} A^{*}$-Sm $C^{*}$ transition. As shown in fig. 3 , the experimental results agree with those predicted by our model in spite of even using simplified $\phi$ functions. It shows also clear differences between the two models; $\Delta \epsilon_{G}, f_{G}$ and $\left\langle P_{E}\right\rangle$ change suddenly at $E_{c}$ in the diverging pitch model, while those in the fixed pitch model these change gradually. Moreover, additional threshold voltage $E_{t}$ where the unwound part of the helix just appears, which we cannot find in the diverging pitch model, is found experimentally and explained by the fixed pitch model. The dielectric response for the Goldstone mode and the effective polarization with dc bias voltage is also explained. According to fig. $3, E_{c}$ found in the diverging pitch model is underestimated. This can be easily understood; in this model, the completely unwound part (U) shown in fig. 1 is not allowed, and $E_{c}$ is the same as the field, at which the U part could appear for very large values of helical pitch. However, if the pitch does not diverge continuously, the U part should appear, and the completely unwound state of helix can be obtained at a much larger field than $E_{c}$ predicted in the diverging pitch 
model, and $E_{c}$ may not be a distinctly defined field, since the physical parameters reach saturated values gradually.

The model for dielectric response during the helical unwinding process suggested in this letter is not complete, because it does not consider the helical fractures in the calculations. The helical fracture may enhance the appearance of the $U$ part and expand its length, and hence, at the field at which the helical fracture occurs, the physical properties may also change sharply. However, the mechanism for the occurrence of the helical fracture is not clear, and it is difficult to estimate the electric field required to generate helical fractures at this state. As reported in our previous paper [9], we observed that the electric field, at which the helical fracture occurs, varies according to the enantiomeric excess ratio in the racemized mixtures. This implies that the material parameters such as the spontaneous polarization, pitch and the interlayer interactions might affect the emergence of the helical fracture. The complexity in the unwinding process of helix in ferroelectric materials, in particular those having large spontaneous polarization, has been reported. Haase et al. [21] suggested a modulated helical structure caused by the tendency of the spontaneous polarization to compensate itself, and Pikin et al. [22] reported dislocation walls induced by moderate electric fields in ferroelectric liquid crystals having large spontaneous polarization. Their interpretations for their observations are different from the helical fracture; the modulated helical structure by Haase et al. [21] uses the continuum approximation, and the dislocation walls by Pikin et al. [22] are apparently different from the $\pi$ walls occurring in smectic layers without significant deformation of the smectic layers themselves. However, their observations imply that the large spontaneous polarization might also play a role in generating the helical fractures. For complete understanding the helical unwinding, the emergence and the propagation of the solitary wave propagation should be investigated to find the mechanism.

As pointed out by Krueger et al., the pitch divergence is interrupted by the surface effects and by the slow and weak diverging force $[6,11,13]$, hence the ideal pitch divergence may be possible only under rather limited experimental and material conditions, though the phenomenon of pitch divergence surely occurs as reported in an earlier paper [12]. Therefore under normal conditions, the fixed pitch model with a broken continuity of the helix is reasonable. The helical fractures are observed under a polarizing microscope. The mechanism of the helical unwinding process in the $\mathrm{Sm} C^{*}$ phase is rather similar from a topological point of view to that affected in a DNA structure. The validity of the model is confirmed by a close agreement between the theoretical and the experimental results for the dielectric strength and the frequency of the Goldstone mode, and the effective polarization. In the diverging pitch model, the critical field $E_{c}$ for a complete unwinding is accompanied with sharp changes in the physical properties. However, the fixed pitch model implies that $E_{c}$ may not be a distinct field since the physical parameters reach saturated values gradually. Electro-optical measurements for the unwinding process support it [9]. In the future, we need to extend this model to include the non-homogeneous domain wall propagation.

We thank SFI grant (02/IN.1/I.031) for funding the work. JKS thanks Samsung Electronics Co. for leave of absence from Seoul.

\section{REFERENCES}

[1] Champoux J. J., Annu. Rev. Biochem., 70 (2001) 369.

[2] Meyer R. B., Mol. Cryst. Liq. Cryst., 40 (1977) 33.

[3] Meyer R. B., Appl. Phys. Lett., 12 (1968) 281.

[4] De Gennes P. G. and Prost J., The Physics of Liquid Crystals (Clarendon Press, Oxford) 1993, p. 385.

[5] Urbanc B. K., Zeks B. and Carlsson T., Ferroelectrics, 113 (1991) 219.

[6] Urbanc B. K. and Zeks B., Phys. Rev. E, 52 (1995) 3892.

[7] Baytch N., Selinger R. L. B., Selinger J. V. and Shashidhar R., Phys. Rev. E, 68 (2003) 041702.

[8] Largerwall S. T., Ferroelectrics, 301 (2004) 15.

[9] Song J. K., Vij J. K. and Kobayashi I., Phys. Rev. E, 75 (2007) 051705.

[10] Song J. K., Fukuda A. and Vij J. K., Phys. Rev. E, 76 (2007) 011708.

[11] Krueger M. and Giesselmann F., J. Appl. Phys., 101 (2007) 094102.

[12] Rozanski S. A. and Kuczynski W., Chem. Phys. Lett., 105 (1984) 104.

[13] Glogarova M., Fousek J., Lejcek L. and Pavel J., Ferroelectrics, 58 (1984) 161.

[14] Pfeiffer M., Wrobel S., Beresnev L. A. and HaAse W., Mol. Cryst. Liq. Cryst., 202 (1991) 193.

[15] Xu H., ViJ J. K., Rappaport A. and Clark N. A., Phys. Rev. Lett., 79 (1997) 249.

[16] Kundu S. K., Suzuki K. and Chaudhuri B. K., Ferroelectrics, 287 (2003) 47.

[17] Roy S. S., Kundu S., Ghosh N., Majumder T. P. and Roy S. K., Mol. Cryst. Liq. Cryst., 328 (1999) 161.

[18] Lagerwall J., Rudquist P., Largerwall S. and Stebler B., Ferroelectrics, 277 (2002) 239.

[19] Orihara H. and Ishibashi Y., Jpn. J. Appl. Phys., 29 (1990) L115.

[20] Ruth J., Selinger J. V. and Shashidhar R., Appl. Phys. Lett., 65 (1994) 1590.

[21] HaAse W., Hiller S., Pfeiffer M. and Beresnev L. A., Ferroelectrics, 140 (1993) 37.

[22] Pikin S. A., Blinov A. M., Beresnev L. A., Hiller S., Schumacher E., Ostrovsky B. I. and HaAse W., Ferroelectrics, 178 (1996) 111. 